\title{
Cosmological post-Newtonian equations from nonlinear perturbation theory
}

\author{
Hyerim Noh \\ Korea Astronomy and Space Science Institute, Daejeon 305-348, Republic of Korea \\ E-mail: hr@kasi.re.kr \\ Jai-chan Hwang \\ Department of Astronomy and Atmospheric Sciences, Kyungpook National \\ University, Daegu 702-701, Republic of Korea \\ E-mail: jchan@knu.ac.kr
}

\begin{abstract}
.
We derive the basic equations of the cosmological first-order post-Newtonian approximation from the recently formulated fully nonlinear and exact cosmological perturbation theory in Einstein's gravity. Apparently the latter, being exact, should include the former, and here we use this fact as a new derivation of the former. The complete sets of equations in both approaches are presented without fixing the temporal gauge conditions so that we can use the gauge choice as an advantage. Comparisons between the two approaches are made. Both are potentially important in handling relativistic aspects of nonlinear processes occurring in cosmological structure formation. We consider an ideal fluid and include the cosmological constant.
\end{abstract}

\section{Contents}

1 Introduction $\quad 1$

2 Exact and fully nonlinear perturbation theory 3

\begin{tabular}{ll|l}
3 & $1 \mathrm{PN}$ approximation & 7
\end{tabular}

$4 \quad$ OPN approximation $\quad 12$

\begin{tabular}{lll}
5 & Discussion & 13 \\
\hline
\end{tabular}

\section{Introduction}

The origin and evolution of the large-scale cosmic structures are important in current scientific cosmology providing links between theories and observations. Many of the important constraints on the cosmological models come from matching observations of 
the large-scale galaxy distribution and motion, and the cosmic microwave background radiation with the theories based on the cosmological perturbation theory.

There are plenty of cosmological situations where the relativistic nature of Einstein's gravity becomes potentially important. Besides its geometric nature involving dynamic space and time, the heavy nonlinear nature of Einstein's gravity also has limited wide applications of the theory in the later nonlinear evolution stage of structures in cosmology. Nonlinear aspects of clustering process of the large-scale structure are mainly studied in the Newtonian context [1, 2, 3, 4, 5] except that the background evolution is provided by Friedmann equations based on Einstein's gravity often with the cosmological constant. Up to the leading-order nonlinear perturbation in Einstein's gravity the success of Newtonian theory is quite remarkable though [6, 7, 8].

Recently we notice advents of two new methods in handling the relativistic and nonlinear aspects of Einstein's gravity in the Friedmann background cosmology. One is the post-Newtonian (PN) approximation [9] with previous studies [10, 11, 12, 13, 14, 15, 16, 17, 18. The other one is the fully nonlinear and exact perturbation theory in Einstein's gravity [19].

In comparison to the perturbation approach (Section 2) which is weakly nonlinear but fully relativistic, the $1 \mathrm{PN}$ approximation (Section 3) can be regarded as fully nonlinear but weakly relativistic. Thus, the two approaches are complementary in understanding the relativistic nonlinear evolution stage of the cosmological structures.

In cosmological situations where the nonlinearity as well as relativistic effects are important we may need full-blown numerical relativity implemented in cosmology. Such a general relativistic numerical simulation in cosmology is not currently available yet. The nonlinear perturbation analysis, being based on perturbative approach, is not sufficient to handle the genuine nonlinear aspects of structure formation accompanied with self-organization and spontaneous formation of structures. In order to handle the relativistic nonlinear process in cosmology we anticipate the PN approach is currently practically suitable to implement in numerical simulation.

Our aim in this work is to derive the cosmological 1PN equations from the nonlinear perturbation theory and to show the relation between the two complementary approaches. The overlap between the 1PN approximation and the linear perturbation theory was studied in [20]. Equations of 1PN approximation, however, contain perturbations up to the fourth order. As the equations of fully nonlinear perturbation theory are exact without even decomposition into background and perturbation, apparently the perturbation theory should include the $1 \mathrm{PN}$ approximation. In this work we will derive the $1 \mathrm{PN}$ equations from the nonlinear perturbation theory equations. In our way of showing the relation we will present the complete sets of equations, the gauge strategies, and solution logics of the two approaches. 


\section{Exact and fully nonlinear perturbation theory}

The perturbation theory assumes all perturbation variables are small and makes perturbation expansion in those variables. The relativistic perturbation theory considers fully relativistic situation, thus applicable in all scales including the superhorizon scale, and in the early universe where radiation and other relativistic components have important roles. In Einstein's gravity the linear perturbation theory has important roles in explaining the origin and evolution of large-scale structures [21, 22, 23, 24, 25, 26, 27, 28]. Recently we have formulated a fully nonlinear and exact perturbation theory [19]. In [19] the exact perturbation equations are also presented without fixing the temporal gauge condition, thus again in a sort of a gauge-ready form, see below.

Our metric convention in the perturbation theory is [27, 19]

$$
d s^{2}=-(1+2 \alpha) c^{2} d t^{2}-2 \chi_{i} c d t d x^{i}+a^{2}(1+2 \varphi) \gamma_{i j} d x^{i} d x^{j},
$$

where spatial index of $\chi_{i}$ is raised and lowered by $\gamma_{i j}$ as the metric; $\gamma_{i j}$ is the comoving part of spatial metric of the Robertson-Walker spacetime; as we consider a spatially flat background in the absence of perturbation, we have $\gamma_{i j}=\delta_{i j}$. Here we assume $a$ to be a function of $t$ only, and $\alpha, \varphi$ and $\chi_{i}$ are arbitrary functions of spacetime. The spatial part of the metric is simple because we already have taken the spatial gauge condition without losing any generality to the fully nonlinear perturbation orders [27, 19] and have ignored the transverse-tracefree tensor-type perturbation. Our spatial gauge (congruence) choice is unique in the sense that under our spatial gauge condition the remaining variables are free from the spatial gauge mode even to the nonlinear order perturbations [19]; any alternative choice leaves the remnant spatial gauge mode even after imposing the spatial gauge condition which should be carefully handled even in the linear order perturbation. In our spatial gauge together with one of the fundamental temporal gauge (slicing) conditions to be suggested below, all the remaining perturbation variables become free from the gauge mode and can be regarded as gauge-invariant ones even to the nonlinear perturbation order [19].

It is important to neglect the transverse-tracfree part of the metric to have the fully nonlinear perturbation formulation in [19]. This is the main assumption restricting the potential applications of the formulation. We may still call our formulation exact and fully nonlinear because, except for ignoring the transverse-tracefree part, in the basic set of equations we have not imposed any condition on the amplitude of the perturbation variables, and also we have not separated the background and perturbation. Ignoring the transverse-tracefree part of the perturbation is consistent with the $1 \mathrm{PN}$ approximation because gravitational waves are known to show up from the $2.5 \mathrm{PN}$ order [29]. The transvere-tracefree part, however, can always be handled perturbatively to any desired nonlinear perturbation order; in perturbation theory, the scalar- and vector-type perturbations can work as sources to the gravitational waves from the second order, see Section 8 in [19]. 
The energy-momentum tensor of an ideal fluid is

$$
\widetilde{T}_{a b}=\widetilde{\mu} \widetilde{u}_{a} \widetilde{u}_{b}+\widetilde{p}\left(\widetilde{u}_{a} \widetilde{u}_{b}+\widetilde{g}_{a b}\right),
$$

where tildes indicate covariant quantities; $\widetilde{\mu}$ and $\widetilde{p}$ are the covariant energy density and pressure, respectively, and $\widetilde{u}_{a}$ is the normalized fluid four-vector [30, 31, 32. In the perturbation theory we may introduce

$$
\widetilde{\mu}=\mu+\delta \mu, \quad \widetilde{p}=p+\delta p, \quad \widetilde{u}_{i} \equiv a \frac{v_{i}}{c},
$$

where the index of $v_{i}$ is raised and lowered by $\gamma_{i j}$ as the metric. In the following we will keep $\widetilde{\mu}$ and $\widetilde{p}$ without decomposition.

In [19] we also have introduced several different definitions of the fluid three-velocity. We introduce the coordinate fluid three-velocity $\bar{v}^{i}$ as

$$
\frac{1}{a} \frac{\bar{v}^{i}}{c} \equiv \frac{\widetilde{u}^{i}}{\widetilde{u}^{0}}
$$

where the index of $\bar{v}^{i}$ is raised and lowered by $\gamma_{i j}$ as the metric; this definition coincides with the one used in our PN study in [9], see equation (20). Compared with $v_{i}$ we have [19]

$$
v_{i}=\frac{\widehat{\gamma}}{\mathcal{N}}\left[(1+2 \varphi) \bar{v}_{i}-\frac{c}{a} \chi_{i}\right] \equiv \widehat{\gamma} \widehat{v}_{i},
$$

where $\widehat{\gamma}$ is the Lorentz factor

$$
\begin{aligned}
\widehat{\gamma}= & \sqrt{1+\frac{v^{k} v_{k}}{c^{2}(1+2 \varphi)}}=\frac{1}{\sqrt{1-\frac{\widehat{\widehat{v}}^{k} \widehat{v}_{k}}{c^{2}(1+2 \varphi)}}} \\
= & \frac{1}{\sqrt{1-\frac{1+2 \varphi}{\mathcal{N}^{2}}\left(\frac{\bar{v}^{k}}{c}-\frac{\chi^{k}}{a(1+2 \varphi)}\right)\left(\frac{\bar{v}_{k}}{c}-\frac{\chi_{k}}{a(1+2 \varphi)}\right)}},
\end{aligned}
$$

and $\mathcal{N}$, related to the lapse function $(N \equiv a \mathcal{N})$, is introduced in equation (16); $\widehat{v}_{i}$ is the fluid three-velocity measured by the Eulerian observer; see the Appendix D of [19]. Although mathematically equivalent, in this work we will use $\bar{v}_{i}$.

We can decompose $\chi_{i}, v_{i}$ and $\bar{v}_{i}$ to scalar- and vector-type perturbations even to nonlinear perturbation orders as

$$
\chi_{i} \equiv c \chi_{, i}+\chi_{i}^{(v)}, \quad v_{i} \equiv-v_{, i}+v_{i}^{(v)}, \quad \bar{v}_{i} \equiv-\bar{v}_{, i}+\bar{v}_{i}^{(v)},
$$

with the vector-type perturbations satisfying $\chi_{i}^{(v) \mid i} \equiv 0$ and $v_{i}^{(v) \mid i} \equiv 0 \equiv \bar{v}_{i}^{(v) \mid i}$; a vertical bar indicates the covariant derivative based on $\gamma_{i j}$ as the metric, thus the same as an ordinary derivative in our present case. Due to the nonlinear relation between $v_{i}$ and $\bar{v}_{i}$ the scalar- and vector-decompositions for $v_{i}$ and $\bar{v}_{i}$ do not coincide with each other to the nonlinear order. To the nonlinear order our scalar- and vector-type perturbations are coupled in the equation level.

In order to match with our convention in the PN approach, here we introduce the dimensions as the following

$$
\begin{aligned}
& {[a]=\left[\gamma_{i j}\right]=\left[\widetilde{g}_{a b}\right]=\left[\widetilde{u}_{a}\right]=1, \quad\left[x^{i}\right]=L, \quad[\alpha]=[\varphi]=\left[\chi^{i}\right]=\left[v^{i} / c\right]=\left[\bar{v}^{i} / c\right]=1,} \\
& {[\kappa]=T^{-1}, \quad[\chi]=T, \quad[v / c]=L, \quad\left[\widetilde{T}_{a b}\right]=[\widetilde{p}]=[\widetilde{\mu}], \quad\left[G \widetilde{\mu} / c^{2}\right]=T^{-2},}
\end{aligned}
$$


where $\kappa$ is the perturbed part of the trace of extrinsic curvature, see equation (9).

The exact and fully nonlinear perturbation equations, without taking the temporal gauge (slicing or hypersurface) condition, are the following [19].

Definition of $\kappa$ :

$\kappa \equiv 3 \frac{\dot{a}}{a}\left(1-\frac{1}{\mathcal{N}}\right)-\frac{1}{\mathcal{N}(1+2 \varphi)}\left[3 \dot{\varphi}+\frac{c}{a^{2}}\left(\chi_{, k}^{k}+\frac{\chi^{k} \varphi_{, k}}{1+2 \varphi}\right)\right]$.

ADM energy constraint:

$$
\begin{aligned}
& -\frac{3}{2}\left(\frac{\dot{a}^{2}}{a^{2}}-\frac{8 \pi G}{3 c^{2}} \widetilde{\mu}-\frac{\Lambda c^{2}}{3}\right)+\frac{\dot{a}}{a} \kappa+\frac{c^{2} \Delta \varphi}{a^{2}(1+2 \varphi)^{2}}=\frac{1}{6} \kappa^{2}-\frac{4 \pi G}{c^{2}}(\widetilde{\mu}+\widetilde{p})\left(\widehat{\gamma}^{2}-1\right) \\
& \quad+\frac{3}{2} \frac{c^{2} \varphi^{, i} \varphi_{, i}}{a^{2}(1+2 \varphi)^{3}}-\frac{c^{2}}{4} \bar{K}_{j}^{i} \bar{K}_{i}^{j} .
\end{aligned}
$$

ADM momentum constraint:

$$
\begin{aligned}
\frac{2}{3} \kappa_{, i} & +\frac{c}{2 a^{2} \mathcal{N}(1+2 \varphi)}\left(\Delta \chi_{i}+\frac{1}{3} \chi_{, i k}^{k}\right)+\frac{8 \pi G}{c^{4}}(\widetilde{\mu}+\widetilde{p}) \frac{a \widehat{\gamma}^{2}}{\mathcal{N}}\left[(1+2 \varphi) \bar{v}_{i}-\frac{c}{a} \chi_{i}\right] \\
& =\frac{c}{a^{2} \mathcal{N}(1+2 \varphi)}\left\{\left(\frac{\mathcal{N}_{, j}}{\mathcal{N}}-\frac{\varphi_{, j}}{1+2 \varphi}\right)\left[\frac{1}{2}\left(\chi_{, i}^{j}+\chi_{i}^{, j}\right)-\frac{1}{3} \delta_{i}^{j} \chi_{, k}^{k}\right]\right. \\
& \left.-\frac{\varphi^{, j}}{(1+2 \varphi)^{2}}\left(\chi_{i} \varphi_{, j}+\frac{1}{3} \chi_{j} \varphi_{, i}\right)+\frac{\mathcal{N}}{1+2 \varphi} \nabla_{j}\left[\frac{1}{\mathcal{N}}\left(\chi^{j} \varphi_{, i}+\chi_{i} \varphi^{, j}-\frac{2}{3} \delta_{i}^{j} \chi^{k} \varphi_{, k}\right)\right]\right\}
\end{aligned}
$$

Trace of ADM propagation:

$$
\begin{aligned}
-3 & \frac{1}{\mathcal{N}}\left(\frac{\dot{a}}{a}\right)^{\cdot}-3 \frac{\dot{a}^{2}}{a^{2}}-\frac{4 \pi G}{c^{2}}(\widetilde{\mu}+3 \widetilde{p})+\Lambda c^{2}+\frac{1}{\mathcal{N}} \dot{\kappa}+2 \frac{\dot{a}}{a} \kappa+\frac{c^{2} \Delta \mathcal{N}}{a^{2} \mathcal{N}(1+2 \varphi)} \\
& =\frac{1}{3} \kappa^{2}+\frac{8 \pi G}{c^{2}}(\widetilde{\mu}+\widetilde{p})\left(\widehat{\gamma}^{2}-1\right)-\frac{c}{a^{2} \mathcal{N}(1+2 \varphi)}\left(\chi^{i} \kappa_{, i}+c \frac{\varphi^{, i} \mathcal{N}_{, i}}{1+2 \varphi}\right)+c^{2} \bar{K}_{j}^{i} \bar{K}_{i}^{j} .
\end{aligned}
$$

Tracefree ADM propagation:

$$
\begin{aligned}
\left(\frac{1}{\mathcal{N}}\right. & \left.\frac{\partial}{\partial t}+3 \frac{\dot{a}}{a}-\kappa+\frac{c \chi^{k}}{a^{2} \mathcal{N}(1+2 \varphi)} \nabla_{k}\right)\left\{\frac{c}{a^{2} \mathcal{N}(1+2 \varphi)}\right. \\
& \left.\times\left[\frac{1}{2}\left(\chi_{, j}^{i}+\chi_{j}^{, i}\right)-\frac{1}{3} \delta_{j}^{i} \chi_{, k}^{k}-\frac{1}{1+2 \varphi}\left(\chi^{i} \varphi_{, j}+\chi_{j} \varphi^{, i}-\frac{2}{3} \delta_{j}^{i} \chi^{k} \varphi_{, k}\right)\right]\right\} \\
& -\frac{c^{2}}{a^{2}(1+2 \varphi)}\left[\frac{1}{1+2 \varphi}\left(\nabla^{i} \nabla_{j}-\frac{1}{3} \delta_{j}^{i} \Delta\right) \varphi+\frac{1}{\mathcal{N}}\left(\nabla^{i} \nabla_{j}-\frac{1}{3} \delta_{j}^{i} \Delta\right) \mathcal{N}\right] \\
& =\frac{8 \pi G}{c^{2}}(\widetilde{\mu}+\widetilde{p})\left[(1+2 \varphi) \frac{\widehat{\gamma}^{2}}{c^{2} \mathcal{N}^{2}}\left(\bar{v}^{i}-\frac{c \chi^{i}}{a(1+2 \varphi)}\right)\left(\bar{v}_{j}-\frac{c \chi_{j}}{a(1+2 \varphi)}\right)\right. \\
& \left.-\frac{1}{3} \delta_{j}^{i}\left(\widehat{\gamma}^{2}-1\right)\right]+\frac{c^{2}}{a^{4} \mathcal{N}^{2}(1+2 \varphi)^{2}}\left[\frac{1}{2}\left(\chi^{i, k} \chi_{j, k}-\chi_{k, j} \chi^{k, i}\right)\right. \\
& +\frac{1}{1+2 \varphi}\left(\chi^{k, i} \chi_{k} \varphi, \chi_{, j}-\chi^{i, k} \chi_{j} \varphi_{, k}+\chi_{k, j} \chi^{k} \varphi^{, i}-\chi_{j, k} \chi^{i} \varphi^{, k}\right) \\
& \left.+\frac{2}{(1+2 \varphi)^{2}}\left(\chi^{i} \chi_{j} \varphi^{, k} \varphi_{, k}-\chi^{k} \chi_{k} \varphi^{, i} \varphi_{, j}\right)\right] \\
& -\frac{c^{2}}{a^{2}(1+2 \varphi)^{2}}\left[\frac{3}{1+2 \varphi}\left(\varphi^{, i} \varphi_{, j}-\frac{1}{3} \delta_{j}^{i} \varphi^{, k} \varphi_{, k}\right)+\frac{1}{\mathcal{N}}\left(\varphi^{, i} \mathcal{N}_{, j}+\varphi_{, j} \mathcal{N}^{, i}-\frac{2}{3} \delta_{j}^{i} \varphi^{, k} \mathcal{N}_{, k}\right)\right] .
\end{aligned}
$$


Covariant energy conservation:

$$
\begin{aligned}
\left(\frac{\partial}{\partial t}\right. & \left.+\frac{1}{a} \bar{v}^{k} \nabla_{k}\right) \widetilde{\mu}+(\widetilde{\mu}+\widetilde{p}) \mathcal{N}(3 H-\kappa)=-(\widetilde{\mu}+\widetilde{p})\left[\frac{1}{a}\left(\bar{v}^{k}-\frac{c \chi^{k}}{a(1+2 \varphi)}\right)_{, k}\right. \\
& \left.+\frac{3 \varphi, k}{a(1+2 \varphi)}\left(\bar{v}^{k}-\frac{c \chi^{k}}{a(1+2 \varphi)}\right)+\frac{1}{\hat{\gamma}}\left(\frac{\partial}{\partial t}+\frac{1}{a} \bar{v}^{k} \nabla_{k}\right) \widehat{\gamma}\right] .
\end{aligned}
$$

Covariant momentum conservation:

$$
\begin{aligned}
& \frac{1}{a \widehat{\gamma}}\left(\frac{\partial}{\partial t}+\frac{1}{a} \bar{v}^{k} \nabla_{k}\right)\left\{\frac{a \widehat{\gamma}}{\mathcal{N}}\left[(1+2 \varphi) \bar{v}_{i}-\frac{c}{a} \chi_{i}\right]\right\} \\
& \quad+\frac{1}{\widetilde{\mu}+\widetilde{p}}\left\{\frac{c^{2} \mathcal{N}}{a \widehat{\gamma}^{2}} \widetilde{p}_{, i}+\frac{1}{\mathcal{N}}\left[(1+2 \varphi) \bar{v}_{i}-\frac{c}{a} \chi_{i}\right]\left(\frac{\partial}{\partial t}+\frac{1}{a} \bar{v}^{k} \nabla_{k}\right) \widetilde{p}\right\} \\
& \quad=-\frac{c^{2}}{a} \mathcal{N}_{, i}-\frac{c}{a^{2} \mathcal{N}}\left[(1+2 \varphi) \bar{v}^{k}-\frac{c}{a} \chi^{k}\right]\left(\frac{\chi_{k}}{1+2 \varphi}\right)_{, i}+\left(1-\frac{1}{\widehat{\gamma}^{2}}\right) \frac{c^{2} \mathcal{N} \varphi, i}{a(1+2 \varphi)}
\end{aligned}
$$

These were derived in Section 3 of [19]; here we use $\bar{v}_{i}$ as the fluid three-velocity instead of $v_{i}$ used in [19] or $\widehat{v}_{i}$ used in [33]. With $\mathcal{N}$ and $\bar{K}_{j}^{i} \bar{K}_{i}^{j}$ given as

$$
\begin{aligned}
& \mathcal{N} \equiv \sqrt{1+2 \alpha+\frac{\chi^{k} \chi_{k}}{a^{2}(1+2 \varphi)}}, \quad \bar{K}_{j}^{i} \bar{K}_{i}^{j}=\frac{1}{a^{4} \mathcal{N}^{2}(1+2 \varphi)^{2}}\left\{\frac{1}{2} \chi^{i, j}\left(\chi_{i, j}+\chi_{j, i}\right)-\frac{1}{3} \chi_{, i}^{i} \chi_{, j}^{j}\right. \\
& \left.-\frac{4}{1+2 \varphi}\left[\frac{1}{2} \chi^{i} \varphi^{, j}\left(\chi_{i, j}+\chi_{j, i}\right)-\frac{1}{3} \chi_{, i}^{i} \chi^{j} \varphi_{, j}\right]+\frac{2}{(1+2 \varphi)^{2}}\left(\chi^{i} \chi_{i} \varphi^{, j} \varphi_{, j}+\frac{1}{3} \chi^{i} \chi^{j} \varphi_{, i} \varphi_{, j}\right)\right\},
\end{aligned}
$$

equations (9)-(15) are the complete set of exact and fully nonlinear perturbation equations valid for the scalar- and vector-type perturbations assuming an ideal fluid in a flat background; $\Lambda$ is the cosmological constant. Notice that we have not separated the background order equations. We only have assumed that $a$ is a function of $t$. In this sense the above set of equations is exact.

In the above set of equations we have not taken the temporal gauge condition yet. As the temporal gauge condition we can impose any one of the following conditions [19]

$$
\begin{array}{ll}
\text { comoving gauge : } & \widehat{v} \equiv 0 \text { or } v \equiv 0, \\
\text { zero-shear gauge : } & \chi \equiv 0, \\
\text { uniform-curvature gauge : } & \varphi \equiv 0, \\
\text { uniform-expansion gauge : } & \kappa \equiv 0, \\
\text { uniform-density gauge : } & \delta \equiv 0,
\end{array}
$$

or combinations of these to all perturbation orders; we can also impose different gauge conditions to different perturbation orders. With the imposition of any one of these slicing conditions the remaining perturbation variables are free from the remnant (spatial and temporal) gauge mode, and have unique gauge-invariant combinations even to the nonlinear order [27, 34, 19].

As long as we take perturbation approach the gauge issues (gauge transformation properties and gauge-invariant combinations) can be handled order by order to any 
nonlinear order (i.e., to the fully nonlinear order). For example, by decomposing the perturbation variable as $\chi \equiv \chi^{(1)}+\chi^{(2)}+\chi^{(3)}+\ldots$ with the numerical indices inside the parenthesis indicating the perturbation orders, the zero-shear gauge can be imposed as $\chi^{(i)}=0$ to each perturbation order to the fully nonlinear order or equivalently $\chi=0$ as an exact zero-shear condition. The gauge issues to the nonlinear order perturbations in concrete forms are presented in Section VI.C of [34] and Section 2 of [19].

It is important to notice that the comoving gauge condition is imposed on $v_{i}$ or $\widehat{v}_{i}$. Even to the linear order, under our congruence (spatial gauge) condition, $\bar{v}$ is slicing (temporal gauge) condition independent; to the linear order we have $\bar{v}=v-\chi / a \equiv v_{\chi}$ which is already gauge-invariant (under our congruence condition). To the nonlinear order we can regard $\widehat{v} \equiv 0$ or $v \equiv 0$ as the comoving gauge condition. For pure scalartype perturbation we have $\widehat{v}=0$ implies $v=0$ and vice versa; in the presence of vector-type perturbation, $\widehat{v}=0$ differs from $v=0$ from the third order in perturbation [19].

\section{3. $1 \mathrm{PN}$ approximation}

The PN approach abandons the geometric spirit of Einstein's gravity and provides the relativistic effects as correction terms in the well known Newtonian equations. That is, the PN approach recovers the concept of absolute space and absolute time. In this way it provides the relativistic effects in the forms of correction terms to the well known Newtonian equations, thus enabling us to use simpler conventional (numerical) treatment. The corrections are made based on an expansion in the dimensionless quantity $\frac{G M}{R c^{2}}$ which is of the same order as $\frac{v^{2}}{c^{2}}$ in motions supported by gravity; $M$, $R$ and $v$ are characteristic mass, dimension and velocity, respectively, of the system we are considering. The PN order $n$ is the same as expansion up to $\left(\frac{G M}{R c^{2}}\right)^{n} \sim\left(\frac{v}{c}\right)^{2 n}$. The PN equations are applicable to weakly relativistic situation with $\frac{G M}{R c^{2}} \ll 1$, and in the subhorizon scale, but are fully nonlinear.

In this work we will consider the first-order PN (1PN) approximation with $n=1$. Chandrasekhar [35] has derived the $1 \mathrm{PN}$ hydrodynamic equations in the Minkowski background in certain gauge condition, see equation (36). In [9] we have derived 1PN hydrodynamic equations in the cosmological background in a gauge-ready form where the temporal gauge condition is left (unfixed) as an option for later use, see equation (35). In [9] we considered a fluid with general pressure, anisotropic stress and flux; we also have considered the presence of cosmological constant and have shown that the proper 1PN approximation demands the flat background. In this work we ignore the anisotropic stress and the flux terms. Our aim in this section is to re-derive the $1 \mathrm{PN}$ hydrodynamic equations from the nonlinear perturbation equations summarized in the previous section. Here we closely follow the conventions used in our 1PN formulation [9].

To the 1PN order Chandrasekhar's metric convention adopted to the cosmological 
background is [35, 36, 9]

$d s^{2}=-\left[1-\frac{1}{c^{2}} 2 U+\frac{1}{c^{4}}\left(2 U^{2}-4 \Phi\right)\right] c^{2} d t^{2}-\frac{1}{c^{3}} 2 a P_{i} c d t d x^{i}+a^{2}\left(1+\frac{1}{c^{2}} 2 V\right) \gamma_{i j} d x^{i} d x^{j}$,

where the spatial index of $P_{i}$ is raised and lowered by $\gamma_{i j}$, the comoving spatial part of the Robertson-Walker metric; as we consider a spatially flat background, we may set $\gamma_{i j}=\delta_{i j}$. Here, $a(t)$ can be regarded as the cosmic scale factor. Similarly as in the metric of perturbation theory in equation (1), in the $\widetilde{g}_{i j}$ part we already have taken spatial gauge conditions without losing any generality, and have ignored the tensor-type perturbation: see Section 6 in [9]. Comparing the two metrics in equations (11) and (18), to the $1 \mathrm{PN}$ order we can identify

$$
\alpha=-\frac{1}{c^{2}}\left[U-\frac{1}{c^{2}}\left(U^{2}-2 \Phi\right)\right], \quad \varphi=\frac{1}{c^{2}} V, \quad \chi_{i}=\frac{1}{c^{3}} a P_{i} .
$$

The 1PN energy-momentum tensor is presented in equation (21) of [9]. With vanishing flux and anisotropic stress, the energy-momentum tensor is given in equation (2). Comparing the energy-momentum tensors and the four-vectors in the two formulations, to the $1 \mathrm{PN}$ order we can identify

$$
\widetilde{\mu} \equiv \widetilde{\varrho} c^{2}\left(1+\frac{1}{c^{2}} \widetilde{\Pi}\right), \quad \bar{v}^{i} \equiv \mathbf{v}, \quad \widetilde{u}^{i} \equiv \frac{1}{c} \frac{1}{a} \bar{v}^{i} \widetilde{u}^{0},
$$

where $\widetilde{\Pi}$ is associated with the internal energy [35]; $\bar{v}_{i}$ is the same as $v_{i}$ used in [9]. From equation (5) we have

$$
v_{i}=\bar{v}_{i}+\frac{1}{c^{2}}\left[\bar{v}_{i}\left(\frac{1}{2} \bar{v}^{2}+U+2 V\right)-P_{i}\right]=\left(1+\frac{1}{c^{2}} \frac{1}{2} \widehat{v}^{2}\right) \widehat{v}_{i},
$$

where $\bar{v}^{2} \equiv \bar{v}^{k} \bar{v}_{k}$ and $\widehat{v}^{2} \equiv \widehat{v}^{k} \widehat{v}_{k}$. The dimensions are the following

$$
\begin{aligned}
& {[U]=[V]=[\widetilde{\Pi}]=c^{2}, \quad\left[P_{i}\right]=c^{3}, \quad[\Phi]=c^{4}, \quad\left[v^{i}\right]=\left[\bar{v}^{i}\right]=c,} \\
& {[\widetilde{p}]=\left[\widetilde{\varrho} c^{2}\right], \quad[G \widetilde{\varrho}]=T^{-2} .}
\end{aligned}
$$

Now, using the identifications between the two approaches made in equations (19) and (20), we can derive the $1 \mathrm{PN}$ equations from the nonlinear perturbation equations in (9)-(15). Equation (9) gives

$$
\kappa=-\frac{1}{c^{2}}\left(3 \frac{\dot{a}}{a} U+3 \dot{V}+\frac{1}{a} P_{\mid k}^{k}\right) .
$$

Equation (13) to $1 \mathrm{PN}$ order gives

$$
V=U \text {. }
$$

Using these, from equations (14), (15), (12) and (11), respectively, we can derive

$$
\begin{aligned}
& \frac{1}{a^{3}}\left(a^{3} \widetilde{\varrho}\right)^{\cdot}+\frac{1}{a}\left(\widetilde{\varrho}^{i}\right)_{\mid i}=-\frac{1}{c^{2}}\left[\widetilde{\varrho}\left(\frac{\partial}{\partial t}+\frac{1}{a} \overline{\mathbf{v}} \cdot \nabla\right)\left(\frac{1}{2} \bar{v}^{2}+3 U+\widetilde{\Pi}\right)+\left(3 \frac{\dot{a}}{a}+\frac{1}{a} \nabla \cdot \overline{\mathbf{v}}\right) \widetilde{p}\right], \\
& \frac{1}{a}\left(a \bar{v}_{i}\right)^{\cdot}+\frac{1}{a} \bar{v}_{i \mid k} \bar{v}^{k}-\frac{1}{a} U_{, i}+\frac{1}{a} \frac{\widetilde{p}_{, i}}{\widetilde{\varrho}}=\frac{1}{c^{2}}\left[\frac{1}{a} \bar{v}^{2} U_{, i}+\frac{2}{a}\left(\Phi-U^{2}\right)_{, i}+\frac{1}{a}\left(a P_{i}\right)^{\cdot}\right.
\end{aligned}
$$




$$
\begin{aligned}
& \quad+\frac{1}{a} \bar{v}^{k}\left(P_{i \mid k}-P_{k \mid i}\right)+\frac{1}{a}\left(\bar{v}^{2}+4 U+\widetilde{\Pi}+\frac{\widetilde{p}}{\widetilde{\varrho}}\right) \frac{\widetilde{p}_{, i}}{\widetilde{\varrho}}-\bar{v}_{i}\left(\frac{\partial}{\partial t}+\frac{1}{a} \overline{\mathbf{v}} \cdot \nabla\right)\left(\frac{1}{2} \bar{v}^{2}+3 U\right) \\
& \left.\quad-\bar{v}_{i} \frac{1}{\widetilde{\varrho}}\left(\frac{\partial}{\partial t}+\frac{1}{a} \overline{\mathbf{v}} \cdot \nabla\right) \widetilde{p}\right], \\
& \frac{\Delta}{a^{2}} U+4 \pi G(\varrho \widetilde{\varrho}-\varrho)=-\frac{1}{c^{2}}\left\{\frac{1}{a^{2}}\left[2 \Delta \Phi-2 U \Delta U+\left(a P_{\mid i}^{i}\right)^{\cdot}\right]+3 \ddot{U}+9 \frac{\dot{a}}{a} \dot{U}+6 \frac{\ddot{a}}{a} U\right. \\
& \left.\quad+8 \pi G\left[\widetilde{\varrho} \bar{v}^{2}+\frac{1}{2}(\widetilde{\varrho} \widetilde{\Pi}-\varrho \Pi)+\frac{3}{2}(\widetilde{p}-p)\right]\right\}, \\
& 0=\frac{1}{a^{2}}\left(P_{\mid k i}^{k}-\Delta P_{i}\right)-16 \pi G \widetilde{\varrho} \bar{v}_{i}+\frac{4}{a}\left(\dot{U}+\frac{\dot{a}}{a} U\right)_{, i} .
\end{aligned}
$$

Terms on the left-hand-side provide the Newtonian $(0 \mathrm{PN})$ limit, and the ones in the right-hand-side are $1 \mathrm{PN}$ contributions. Notice that the $1 \mathrm{PN}$ terms include up to fourthorder perturbations.

These are the same as cosmological 1PN equations presented in [9] for vanishing anisotropic stress and flux; in the Minkowski background and under a certain gauge condition, see [35]. These follow from the energy-conservation, momentum-conservation, trace of ADM propagation $\left(\widetilde{G}_{0}^{0}-\widetilde{G}_{i}^{i}=2 \widetilde{R}_{0}^{0}\right)$, and momentum-constraint $\left(\widetilde{G}_{i}^{0}\right)$ equations, respectively; for the general case with the anisotropic stress, see equations (114), (115), (119) and (120) in [9]. In the PN approximation, the energy constraint equation $\left(2 \widetilde{G}_{0}^{0}=\widetilde{R}_{0}^{0}-\widetilde{R}_{i}^{i}\right)$ in equation (10) gives $0 \mathrm{PN}$ part of equation (27); see equation (8) and equations (80) and (87) in [9].

Our cosmological PN approach assumes a flat cosmological background, but is valid in the presence of the cosmological constant. Equations for the background are

$$
\begin{aligned}
& \frac{\ddot{a}}{a}=-\frac{4 \pi G}{3} \varrho\left[1+\frac{1}{c^{2}}\left(\Pi+3 \frac{p}{\varrho}\right)\right]+\frac{\Lambda c^{2}}{3}, \\
& \frac{\dot{a}^{2}}{a^{2}}=\frac{8 \pi G}{3} \varrho\left(1+\frac{1}{c^{2}} \Pi\right)+\frac{\Lambda c^{2}}{3}, \quad \dot{\varrho}=-3 \frac{\dot{a}}{a} \varrho,
\end{aligned}
$$

and $\dot{\mu}+3(\dot{a} / a)(\mu+p)=0$ with $\dot{\Pi}+3(\dot{a} / a) p / \varrho=0$; see Section 4.1 in [9]. These background order equations based on Einstein's gravity were subtracted in deriving the PN equations; see Section 3.2 in [9]. This is related to the fact that self-consistent treatment of cosmological world model is not possible in Newton's gravity. Without a guide by Einstein's gravity [37], the spatially homogeneous and isotropic cosmological world model based on Newton's gravity is known to be incomplete and indeterminate [38, 39].

In order to solve the $1 \mathrm{PN}$ equations it will be convenient to have the equations for $U, \dot{U}$ and $\ddot{U}$ to the $0 \mathrm{PN}$ order [16]. For $U$, equation (27) gives

$$
\frac{\Delta}{a^{2}} U=-4 \pi G(\widetilde{\varrho}-\varrho) \text {, }
$$

valid to the $0 \mathrm{PN}$ order. By taking a divergence of equation (28), and using equation (27), we have

$$
\frac{\Delta}{a^{2}} \dot{U}=4 \pi G\left[\frac{\dot{a}}{a}(\widetilde{\varrho}-\varrho)+\frac{1}{a}\left(\widetilde{\varrho} \bar{v}^{i}\right)_{\mid i}\right],
$$


valid to the $0 \mathrm{PN}$ order. By taking a time derivative, and using equations (25)-(27), we have

$\frac{\Delta}{a^{2}} \ddot{U}=4 \pi G\left\{\left(\frac{\ddot{a}}{a}-2 \frac{\dot{a}^{2}}{a^{2}}\right)(\widetilde{\varrho}-\varrho)-\frac{1}{a^{2}}\left[4 \dot{a} \widetilde{\varrho} \bar{v}^{i}+\left(\widetilde{\varrho} \bar{v}^{i} \bar{v}^{k}\right)_{\mid k}-\widetilde{\varrho} U^{i}+\widetilde{p}^{, i}\right]_{\mid i}\right\}$,

valid to the $0 \mathrm{PN}$ order.

In the above $1 P N$ equations the spatial gauge conditions were fixed in the same way as in the perturbation theory, but we have not fixed the temporal (slicing) condition yet. The gauge transformation property in the 1PN approximation was thoroughly studied in Section 6 of [9], and in the following we briefly review it. In our 1PN metric convention in equation (18) we already have taken spatial gauge condition by setting $g_{i j}=a^{2}\left(1+c^{-2} 2 V\right) \delta_{i j}$; see Section 6 in [9]. Under the remaining gauge transformation $\widehat{x}^{a}=x^{a}+\xi^{a}\left(x^{e}\right)$ with

$$
\xi^{0}=\frac{1}{c} \xi^{(2) 0}+\frac{1}{c^{3}} \xi^{(4) 0},
$$

we can set $\xi^{(2) 0}=0$ without losing any generality: see equation (173) in [9]. In this case variables $U$ and $V$ are gauge-invariant, and we have [see equations (175) and (176) in [9]

$$
\widehat{P}_{i}=P_{i}-\frac{1}{a} \xi_{, i}^{(4) 0}, \quad \widehat{\Phi}=\Phi+\frac{1}{2} \dot{\xi}^{(4) 0} .
$$

Thus, in the PN approach we have freedom to impose the temporal gauge (slicing or hypersurface) condition on $P_{\mid i}^{i}$ or $\Phi$; fixing $\Phi=0$ in all coordinates leaves the remnant gauge mode $\xi^{(4) 0}(\mathbf{x})$, whereas setting $P^{i}{ }_{i}=0$ in all coordinate as the gauge condition completely removes the gauge mode. A combination $2 \Phi_{, i}+\left(a P_{i}\right)^{*}$ is gauge-invariant. The variables $\widetilde{\varrho}$ and $\widetilde{\Pi}$ are not fixed individually under the gauge transformation, and varies as $\widehat{\widetilde{\varrho}}=\widetilde{\varrho}+\varrho^{(2)} / c^{2}$ and $\widehat{\widetilde{\Pi}}=\widetilde{\Pi}-\varrho^{(2)} / \varrho$ where $\varrho^{(2)}$ is an undetermined transformation function, see equation (192) in [9]. Thus a combination $\widetilde{\varrho}\left(1+\widetilde{\Pi} / c^{2}\right)$ is gauge invariant; $\bar{v}_{i}$ is also gauge invariant: see equation (193) in [9]. Using the above gauge transformation properties we can show that equations (25)-(28) are invariant under the gauge transformation to the appropriate PN orders.

In [9] we have introduced a general temporal gauge condition as

$$
\frac{1}{a} P_{\mid i}^{i}+n \dot{U}+m \frac{\dot{a}}{a} U=0,
$$

where $n$ and $m$ can be arbitrary real numbers. Several temporal gauge conditions used in the literature are

$$
\begin{array}{ll}
\text { Harmonic gauge : } & n=4, m=\text { arbitrary, } \\
\text { Chandrasekhar's gauge : } & n=3, m=\text { arbitrary, } \\
\text { Uniform-expansion gauge : } & n=3=m, \\
\text { Transverse-shear gauge : } & n=0=m .
\end{array}
$$

Compared with the fundamental temporal gauge conditions of the perturbation theory in equation (17), the uniform-expansion gauge is the same as $\kappa=0$ in the perturbation 
theory; the Chandrasekhar's gauge is the same as $\kappa+(m-3)(\dot{a} / a) \varphi=0$ in the perturbation theory which is a fine gauge condition without remnant gauge mode. In the cosmological PN approach, [16] also took $n=3$ as their slicing condition. The transverse-shear gauge is the same as the zero-shear gauge with $\chi=0\left(\right.$ or $\left.\chi_{\mid i}^{i}=0\right)$. The Harmonic gauge condition can be written as $\dot{\alpha}+H \alpha+\kappa=0$ which leaves heavy remnant gauge modes in the perturbation theory even to the linear order (see the Appendix in [40]), but not in the case of $1 \mathrm{PN}$ approximation [9]. In the $\mathrm{PN}$ approximation the comoving gauge $\left(v^{i}{ }_{\mid i}=0\right)$, the uniform-curvature gauge $(\varphi=0)$, and the uniformdensity gauge $(\delta \equiv 0)$ in the perturbation theory, corresponding to $\bar{v}^{i}{ }_{i} \equiv 0, V \equiv 0$, and $\widetilde{\varrho}-\varrho \equiv 0$, respectively, are not available.

Equation (27) shows that the harmonic gauge condition makes the Laplacian operator $\frac{\Delta}{a^{2}}$ in the $0 \mathrm{PN}$ limit replaced by a d'Alembertian operator $\frac{\Delta}{a^{2}}-\frac{\partial^{2}}{c^{2} \partial t^{2}}$ by the $1 \mathrm{PN}$ correction terms, thus making the Poisson's equation in 0PN limit to become a wave equation with the propagation speed $c$ by the 1PN correction. Examination of equation (27) shows that, under the general gauge condition in equation (35), the propagation speed of the gravitational potential $U$ is

$$
\frac{c}{\sqrt{n-3}}
$$

see equation (213) of [9]. Thus, the uniform-expansion gauge and the Chandrasekhar's gauge leave the action-at-a-distance nature of the Poisson's equation, and the transverseshear gauge makes equation (27) to be no longer a wave equation. Although the propagation speed of the gravitational potential depends on the gauge choice, the propagation speed of the (physical) Weyl tensor naturally does not depend on the gauge choice and is always $c$ : see Section 7 of [9]. Exact analogy can be found in the electromagnetism where the propagation speed of the field potential depends on the gauge choice, whereas the the propagation speed of the (physical) field strength is always the speed of light, see [41].

Under the gauge condition in equation (35), equation (28) becomes

$$
\frac{\Delta}{a^{2}} P_{i}=\frac{1}{a}\left[(4-n) \dot{U}+(4-m) \frac{\dot{a}}{a} U\right]_{, i}-16 \pi G \widetilde{\varrho} \bar{v}_{i} .
$$

The temporal gauge condition corresponds to fixing $n$ and $m$. As $U$ is gauge-invariant to $1 \mathrm{PN}$ order, independently of the values of $n$ and $m$, using equation (38) all variables in our set of equations are free from the gauge mode, and can be regarded as gauge invariant.

By introducing a gauge-invariant combination

$$
\mathcal{U}_{, i} \equiv U_{, i}+\frac{1}{c^{2}}\left[2 \Phi_{, i}+\left(a P_{i}\right)^{\cdot}\right]
$$

the $\Phi$ and $\left(a P_{i}\right)^{\cdot}$ terms in the right-hand-sides of equations (26) and (27) can be absorbed to the $U$ terms in the left-hand-sides by replacing $U$ to $\mathcal{U}$. Equations (26) and (27) become

$$
\frac{1}{a}\left(a \bar{v}_{i}\right)^{\cdot}+\frac{1}{a} \bar{v}_{i \mid k} \bar{v}^{k}-\frac{1}{a} \mathcal{U}_{, i}+\frac{1}{a} \frac{\widetilde{p}_{, i}}{\widetilde{\varrho}}=\frac{1}{c^{2}}\left[\frac{1}{a}\left(\bar{v}^{2}-4 U\right) U_{, i}+\frac{2}{a} \bar{v}^{k} P_{[i \mid k]}\right.
$$




$$
\begin{aligned}
& +\frac{1}{a}\left(\bar{v}^{2}+4 U+\widetilde{\Pi}+\frac{\widetilde{p}}{\widetilde{\varrho}}\right) \frac{\widetilde{p}_{, i}}{\widetilde{\varrho}}-\bar{v}_{i}\left(\frac{\partial}{\partial t}+\frac{1}{a} \overline{\mathbf{v}} \cdot \nabla\right)\left(\frac{1}{2} \bar{v}^{2}+3 U\right) \\
& \left.-\bar{v}_{i} \frac{1}{\widetilde{\varrho}}\left(\frac{\partial}{\partial t}+\frac{1}{a} \overline{\mathbf{v}} \cdot \nabla\right) \widetilde{p}\right], \\
\frac{\Delta}{a^{2}} \mathcal{U} & +4 \pi G(\widetilde{\varrho}-\varrho)=-\frac{1}{c^{2}}\left\{3 \ddot{U}+9 \frac{\dot{a}}{a} \dot{U}+6 \frac{\ddot{a}}{a} U\right. \\
& \left.+8 \pi G\left[\widetilde{\varrho} \bar{v}^{2}+(\widetilde{\varrho}-\varrho) U+\frac{1}{2}(\widetilde{\varrho} \widetilde{\Pi}-\varrho \Pi)+\frac{3}{2}(\widetilde{p}-p)\right]\right\} .
\end{aligned}
$$

Analysis can be proceeded as follows. The fluid variables $\widetilde{p}$ and $\widetilde{\Pi}$ should be given by the equation of state according to the system we are modeling; for example, for pressureless dust or cold dark matter, we may set $\widetilde{p}=0=\widetilde{\Pi}$. Choose the gauge condition, thus fixing $n$ and $m$ in equation (38). We can determine $\widetilde{\varrho}, \bar{v}_{i}$ and $\mathcal{U}$ to $1 \mathrm{PN}$ order by solving equations (25), (40) and (41). In order to determine $U, \dot{U}, \ddot{U}$, and $P_{i}$ in the right-hand-sides of these equations to the $0 \mathrm{PN}$ order, we can solve equations (30), (31), (32), and (38) respectively; inverting the Laplacian by volume integration in these equations would be time consuming in numerical implementation. The background evolution should be determined by equation (29) simultaneously.

We may identify cosmological situations where the cosmological PN approach might have important applications. The current cosmological paradigm favors a model where the large-scale structures are in the linear stage, whereas small-scale structures are apparently in fully nonlinear stage. The standard strategy is to assume that the smallscale nonlinear structures can be fully handled by the Newtonian gravity. In the galactic and cluster scales we have the general relativistic measure $\frac{G M}{R c^{2}} \sim \frac{v^{2}}{c^{2}} \sim 10^{-6}-10^{-4}$, thus small but nonvanishing, thus indeed the 1PN (weakly relativistic) assumption is quite sufficiently valid. We believe the $1 \mathrm{PN}$ approach would be relevant to estimate the general relativistic effects in the nonlinear clustering processes of the galaxy cluster-scale and the large-scale structures.

\section{4. $0 P N$ approximation}

We can view the Newtonian limit as the 0PN approximation; see Sec. 4.2 of [9]. Indeed, the $1 \mathrm{PN}$ approximation studied in the previous section properly contains the Newtonian hydrodynamic equations at the OPN level; in the Minkowski background, see [35]. In order to have the Newtonian hydrodynamic equations as the 0PN limit, we should take the $0 \mathrm{PN}$ metric as

$$
d s^{2}=-\left(1-\frac{1}{c^{2}} 2 U\right) c^{2} d t^{2}+a^{2}\left(1+\frac{1}{c^{2}} 2 V\right) \delta_{i j} d x^{i} d x^{j} .
$$

Although $\alpha$ (thus $U$ ) contains the perturbed Newtonian gravitational potential, the full Einstein's equation demands to keep $\varphi$ (thus $V$ ) which can be regarded as the PN order [33], see equation (18). Thus, as the $0 \mathrm{PN}$ limit we identify

$$
\alpha=-\frac{1}{c^{2}} U, \quad \varphi=\frac{1}{c^{2}} V, \quad \chi_{i}=0, \quad \bar{v}_{i}=\mathbf{v},
$$


and take the leading order terms in the $1 / c$ expansion. Equation (13) gives $\varphi=-\alpha$, thus $V=U$. Equation (11) gives

$$
\kappa=-\frac{12 \pi G}{c^{2}} a \Delta^{-1}\left(\widetilde{\varrho} \bar{v}^{i}\right)_{, i},
$$

equation (14), equation (15)), equation (12), and equation (9), respectively, give

$$
\begin{aligned}
& \dot{\tilde{\varrho}}+3 \frac{\dot{a}}{a} \widetilde{\varrho}+\frac{1}{a}\left(\widetilde{\varrho} \bar{v}^{i}\right)_{, i}=0, \\
& \frac{1}{a}\left(a \bar{v}_{i}\right)^{\cdot}+\frac{1}{a} \bar{v}_{i, k} \bar{v}^{k}-\frac{1}{a} U_{, i}+\frac{1}{a \widetilde{\varrho}} \widetilde{p}_{, i}=0, \\
& \frac{\Delta}{a^{2}} U+4 \pi G(\widetilde{\varrho}-\varrho)=0, \\
& \dot{U}+\frac{\dot{a}}{a} U-4 \pi G a \Delta^{-1}\left(\varrho \bar{v}^{i}\right)_{, i}=0 .
\end{aligned}
$$

Equation (12) also gives equation (47). Equation (48) is consistent with equation (28). These are the well known perturbed Newtonian hydrodynamic equations in the cosmological background; see Sections 7-9 of [1].

One may wonder why we need the presence of $V$ term in equation (42) which is ordinarily known as the 1PN order term; it's presence as the 1PN correction is important to have correct light deflection in Einstein's gravity, see equation (149) in [9]. This issue was addressed in the nonlinear perturbation theory context in [33. Here, in our PN approach we clarify the following situation. The hydrodynamic equations and the Poisson's equation in equations (45)-(47) properly follow from equations (14), (15) and (12), respectively, without involving the $\varphi$ (thus $V$ ) term; in this sense the $0 \mathrm{PN}$ metric in $\alpha$ (thus $U$ ) is enough to get the proper Newtonian equation. However, additionally, in order to properly have equation (47) from equation (10), and equation (48) from equation (9) we need the presence of the $\varphi$ (thus $V$ ) term which is ordinarily regarded as the 1PN correction. Therefore, although the full Newtonian equations follow from Einstein's equation without involving the $V$ term, the presence of $V$ term is demanded by the self-consistency of the full Einstein's equation, see [35, 42, 43].

As a complementary study in the perturbation theory, in [33] we have shown that in two gauge conditions (the zero-shear gauge and the uniform-expansion gauge) the fully nonlinear perturbation equations properly reduce to the Newtonian hydrodynamic equations in the infinite speed-of-light limit (weak gravity, slow-motion, negligible pressure and internal energy compared with the mass-energy density, and subhorizon limits). As the two gauge conditions are consistent with the PN approaches, the result is consistent with the present study based on the PN approximation; see below equation (36).

\section{Discussion}

Our main point in this work is a new derivation of the 1PN equations from the recently formulated fully nonlinear and exact perturbation theory. In this way we have 
clarified the relation between the two approaches, nonlinear perturbation theory versus PN approach, which may play key roles in handling relativistic aspects of nonlinear processes in cosmology. In order to clarify the relations between the two approaches we presented some overlapping materials with [9] and [19]. The master equations and gauge strategies in both approaches are presented assuming an ideal fluid in the presence of the cosmological constant.

We anticipate that the fully nonlinear perturbation theory will be useful to derive $2 \mathrm{PN}$ equations as well where the gravitational wave backreaction does not appear; in the Minkowski background and in certain gauge conditions see [36] for 2PN equations, and 29] for 2.5PN equations with first appearance of the radiative-reaction term. Besides ignoring the transverse-tracefree part of the metric (which should be handled perturbatively), one other possible limit of our nonlinear perturbation theory in handling the higher order PN approach is the spatial gauge condition taken leaving the spatial part of the metric as in equation (11). In the perturbation theory our spatial gauge condition is the unique choice without remnant gauge mode to all perturbation orders [27, 34, 19]. Whether the same spatial gauge condition will remain suitable for higherorder PN approximation is the subject for future investigation. Under the current situation where even 1PN equations are not implemented in the cosmological numerical simulation yet, we do not have pressing demand to go for the higher PN effects, though.

We hope that at some point in near future the equations based on these two approaches could be implemented numerically as a step toward the relativistic numerical simulations in cosmology. The PN approach preserves the Newtonian nature of the theory with the Einstein's gravity contributions appearing as the PN correction terms: see equations (25)-(28) together with the gauge condition in (35), or equations (40) and (41) in a gauge-invariant form. Thus, the 1PN equations are easy to implement in the conventional Newtonian hydrodynamic simulation code except that it could be quite time consuming due to the presence of bare potential terms. The 1PN equations will

be important where $\frac{G M}{R c^{2}} \sim \frac{v^{2}}{c^{2}}$ is small but not negligible, and the galaxy clusters and the large-scale cosmic structure satisfy these conditions. Although the correction terms are small, whether the PN corrections could leave long term (secular) signature in the large-scale structure is a subject left for future cosmological PN numerical simulation.

The full-blown numerical relativity is often based on the ADM (Arnowitt-DeserMisner) equations [44]. As the names of equations (91)-(15) indicate, our nonlinear perturbation equations are the same as the ADM equations based on our spatial gauge condition and the perturbation theory notation; we used the covariant conservation equations instead of the ADM conservation equations, but the choice is a matter of convenience and both are presented in [19]; for the ADM conservation equations, see equations (33) and (34) in [19]. Thus, our exact and fully nonlinear perturbation equations can be regarded as exact Einstein's equations adapted to cosmological perturbation theory. Ignoring the transverse-tracefree part of the metric in our formulation is certainly limiting the potential applications, but as demonstrated in [19], the fully nonlinear equations are quite powerful in deriving the higher order 
nonlinear perturbation equations for the scalar- and vector-type perturbations in diverse fundamental temporal gauge (slicing) conditions.

\section{Acknowledgments}

We wish to thank the referee for useful suggestions. J.H. was supported by KRF Grant funded by the Korean Government (KRF-2008-341-C00022). H.N. was supported by grant No. 2012 R1A1A2038497 from NRF.

\section{References}

[1] P.J.E. Peebles, The large-scale structure of the universe, Princeton Univ. Press, Princeton (1980).

[2] Ya.B. Zel'dovich and I.D. Novikov, Relativistic astrophysics, Vol 2, The structure and evolution of the universe, Univ. Chicago Press, Chicago (1983).

[3] E. Bertschinger, Ann. Rev. Astron. Astrophys. 36 (1998) 599.

[4] F. Bernardeau, S. Colombi, E. Gaztanaga and R. Scoccimarro, Phys. Rep. 367 (2002) 1.

[5] J. Kim, C. Park, G. Rossi, S. M. Lee and J.R. III Gott, J. Korean Astron. Soc. 44 (2011) 217.

[6] E.T. Vishniac, Mon. Not. Roy. Astron. Soc. 203 (1983) 345.

[7] J. Hwang and H. Noh, Mon. Not. Roy. Astron. Soc. 367 (2006) 1515.

[8] D. Jeong, J. Gong, H. Noh and J. Hwang, Astrophys. J. 722 (2011) 1.

[9] J. Hwang, H. Noh and D. Puetzfeld, JCAP 03 (2008) 010.

[10] T. Futamase, Phys. Rev. Lett. 61 (1988) 2175.

[11] K. Tomita, Prog. Theor. Phys. 79 (1988) 258.

[12] T. Futamase, Mon. Not. Roy. Astron. Soc. 237 (1989) 187.

[13] K. Tomita, Prog. Theor. Phys. 85 (1991) 1041.

[14] T. Futamase, Prog. Theor. Phys. 86 (1993) 389.

[15] T. Futamase, Prog. Theor. Phys. 89 (1993) 581.

[16] M. Shibata and H. Asada, Prog. Theor. Phys. 94 (1995) 11.

[17] H. Asada and T. Futamase, Prog. Theor. Phys. Suppl. 128 (1997) 123.

[18] M. Takada and T. Futamase, Mon. Not. Roy. Astron. Soc. 306 (1999) 64.

[19] J. Hwang and H. Noh, Mon. Not. Roy. Astron. Soc. in press, (2013), arXiv:1207.0264v2.

[20] H. Noh and J. Hwang, Astrophys. J. 757 (2012) 145.

[21] E.M. Lifshitz, J. Phys. (USSR) 10 (1946) 116.

[22] E.R. Harrison, Rev. Mod. Phys. 39 (1967) 862.

[23] R.K. Sachs and A.M. Wolfe, Astrophys. J. 147 (1967) 73.

[24] G.B. Field and L.C. Shepley, Astrophys. Space. Sci. 1 (1968) 309.

[25] H. Nariai, Prog. Theor. Phys. 41 (1969) 686.

[26] J.M. Bardeen, Phys. Rev. D 22 (1980) 1882.

[27] J.M. Bardeen, Particle Physics and Cosmology, edited by L. Fang and A. Zee, Gordon and Breach, London (1988) 1.

[28] V.F. Mukhanov, Sov. Phys. JETP 68 (1988) 1297.

[29] S. Chandrasekhar and F.P. Esposito, Astrophys. J. 160 (1970) 153.

[30] J. Ehlers, Proceedings of the mathematical-natural science of the Mainz academy of science and literature, Nr. 11 (1961) 792, english translation in Gen. Rel. Grav. 25 (1993) 1225.

[31] G.F.R. Ellis, General relativity and cosmology, Proceedings of the international summer school of physics Enrico Fermi course 47, edited by R. K. Sachs, Academic Press, New York, (1971).

[32] G.F.R. Ellis, Cargese Lectures in Physics, edited by E. Schatzmann, Gordon and Breach, New York (1973).

[33] J. Hwang and H. Noh, JCAP 081 (2013) 0812. 
[34] H. Noh and J. Hwang, Phys. Rev. D 69 (2004) 104011.

[35] S. Chandrasekhar, Astrophys. J. 142 (1965) 1488.

[36] S. Chandrasekhar and Y. Nutku, Astrophys. J. 158 (1969) 55.

[37] A. A. Friedmann, Zeitschrift für Physik 10 (1922) 377, english translation in Gen. Rel. Grav. 31 (1999) 1991.

[38] D. Layzer, Astron. J. 59 (1954) 268.

[39] D.S. Lemons, Am. J. Phys. 56 (1988) 502.

[40] J. Hwang, Astrophys. J. 415 (1993) 486.

[41] J.D. Jackson, Am. J. Phys. 70 (2002) 917.

[42] L. Kofman and D. Pogosyan, Astrophys. J. 442 (1995) 30.

[43] S. Matarrese and D. Terranova, Mon. Not. Roy. Astron. Soc. 283 (1996) 400.

[44] R. Arnowitt, S. Deser and C.W. Misner, Gravitation: an introduction to current research, edited by L. Witten, Wiley, New York (1962) 227. 